\title{
Orthogonal parallel-flats designs for hierarchical models
}

\author{
C.T. Liao \\ Division of Biometry, Institute of Agronomy, National Taiwan University, No. 1, Section 4, Roosevelt Road, \\ Taipei 10764, Taiwan
}

Received September 1998; received in revised form June 1999

\begin{abstract}
Orthogonal designs are very popular in many experimental studies because of their optimality properties, ease of analysis, and ease of interpretation. In this paper, we prove a necessary and sufficient condition for a parallel-flats design to be orthogonal for estimating the factorial effects of hierarchical models for general $s_{1}^{n_{1}} \times s_{2}^{n_{2}} \times \cdots \times s_{g}^{n_{g}}$ mixed factorial experiments, where $s_{1}, s_{2}, \ldots, s_{g}$ are assumed to be distinct primes. Some examples are given to illustrate the results. (C) 2000 Elsevier Science B.V. All rights reserved
\end{abstract}

Keywords: Orthogonal arrays; Fractional factorial designs; Design resolution; Complex factorial effects

\section{Introduction}

The class of parallel-flats designs (PFDs) was first introduced by Connor and Young (1961). Srivastava and Chopra (1973) stated a set of necessary and sufficient conditions for a PFD to be an orthogonal design of resolution $d$ for symmetrical $s^{n}$ factorial experiments. A proof of this result can be found in Srivastava and Throop (1990). A general theory of PFDs was presented in Srivastava et al. (1984) which was further developed in Srivastava (1987). Then Srivastava and Li (1996) obtained a set of necessary and sufficient conditions for a PFD to be an orthogonal design for estimating any arbitrary set of factorial effects. This result was developed for the case of symmetrical $s^{n}$ factorial experiments with $s$ being a prime number. Liao and Iyer (1998) extended their results to $s_{1}^{n_{1}} \times s_{2}^{n_{2}}$ mixed factorial experiments with $s_{1}$ and $s_{2}$ being distinct primes. The results extended to general mixed $s_{1}^{n_{1}} \times s_{2}^{n_{2}} \times \cdots \times s_{g}^{n_{g}}$ factorial experiments, for $g>2$, could be very complicated for considering arbitrary set of factorial effects.

In the next section, we review the definitions of complex factorial effects, orthogonal designs and PFDs. In Section 3, we prove a theorem for a PFD to be orthogonal for hierarchical models for general $s_{1}^{n_{1}} \times s_{2}^{n_{2}} \times \cdots \times s_{g}^{n_{g}}$ mixed factorial experiments, where $s_{1}, s_{2}, \ldots, s_{g}$ are assumed to be distinct primes. We give some examples to demonstrate the main theorem.

\section{Preliminaries}

We first briefly review the definitions of complex factorial effects, orthogonal designs and PFDs. The reader can consult Liao and Iyer (1998) for more details. 


\subsection{Complex factorial effects}

Consider $n$ factors, say $F_{1}, F_{2}, \ldots, F_{n}$, at levels $s_{1}, s_{2}, \ldots, s_{n}$, respectively. $s_{1}, s_{2}, \ldots, s_{n}$ are primes but not necessarily distinct. Define the set of vectors $\Omega$ by

$$
\Omega=\left\{\boldsymbol{p}=\left(p_{1}, p_{2}, \ldots, p_{n}\right) \mid p_{i} \in\left\{0,1, \ldots, s_{i}-1\right\}, \text { for } i=1,2, \ldots, n\right\}
$$

For $\boldsymbol{t}=\left(t_{1}, t_{2}, \ldots, t_{n}\right) \in \Omega$, let $u(\boldsymbol{t})$ denote the expected response when factor $F_{i}$ is at level $t_{i}$ for $i=1,2, \ldots, n$. Also, let $\boldsymbol{u}$ denote the vector of size $s_{1} s_{2} \cdots s_{n}$ whose elements are the $u(t)$ arranged in lexicographic order. Finally, let $\boldsymbol{L}\left(s_{1}, s_{2}, \ldots, s_{n}\right)$ be a square matrix of dimension $s_{1} s_{2} \cdots s_{n}$, whose rows and columns are labeled by elements of $\Omega$ such that, for $\boldsymbol{p}, \boldsymbol{q} \in \Omega$, the element of $\boldsymbol{L}\left(s_{1}, s_{2}, \ldots, s_{n}\right)$ corresponding to row $\boldsymbol{p}$ and column $\boldsymbol{q}$ is defined to be

$$
L_{p q}\left(s_{1}, s_{2}, \ldots, s_{n}\right)=w_{1}^{p_{1} q_{1}} w_{2}^{p_{2} q_{2}} \cdots w_{n}^{p_{n} q_{n}}
$$

where $w_{i}$ is a primitive $s_{i}$ th root of unity. The vector $\boldsymbol{\beta}$ of general complex factorial effects, associated with a $s_{1} \times s_{2} \times \cdots \times s_{n}$ factorial experiment, is defined by

$$
\boldsymbol{\beta}=\boldsymbol{L}^{-1}\left(s_{1}, s_{2}, \ldots, s_{n}\right) \boldsymbol{u}=\frac{1}{s_{1} s_{2} \cdots s_{n}} \overline{\boldsymbol{L}}^{\prime}\left(s_{1}, s_{2}, \ldots, s_{n}\right) \boldsymbol{u},
$$

where $\overline{\boldsymbol{L}}\left(s_{1}, s_{2}, \ldots, s_{n}\right)$ represents the conjugate complex of $\boldsymbol{L}\left(s_{1}, s_{2}, \ldots, s_{n}\right)$. In particular, for $\boldsymbol{e} \in \Omega$,

$$
\beta(\boldsymbol{e})=\frac{1}{s_{1} s_{2} \cdots s_{n}} \sum_{\boldsymbol{t} \in \Omega} \bar{L}_{\boldsymbol{e t}}\left(s_{1}, s_{2}, \ldots, s_{n}\right) u(\boldsymbol{t}) .
$$

This defines a total $s_{1} s_{2} \cdots s_{n}$ factorial effects $\beta(\boldsymbol{e})$, one corresponding to each $\boldsymbol{e}$ in $\Omega$.

$\beta(\boldsymbol{e})$ is said to be a factorial effect belonging to the effects subspace $F_{i_{1}} F_{i_{2}} \ldots F_{i_{k}}$ whenever $\boldsymbol{e}=\left(e_{1}, \ldots, e_{n}\right)$ is a nonzero vector in $\Omega$ such that $e_{i_{r}} \neq 0$ for $r \in\{1,2, \ldots, k\}$ and $e_{i_{r}}=0$ for $r \notin\{1,2, \ldots, k\}$. $\beta(\mathbf{0})$ is the general mean which belongs to a unique one-dimension effect subspace. Thus there are 1 general-mean effect subspace, $n$ main-effect effects subspaces, $n(n-1) / 2$ two-factor-interaction effects subspaces, etc., a total of $2^{n}$ effects subspaces to be defined. Any two effects having nonzero elements in exactly the same positions are in the same effects subspace.

Next, we first define geometric parameters vector. Then we define a geometric parameters vector to be hierarchical.

Definition 2.1. A vector $\boldsymbol{\beta}$ of factorial effects will be called geometric if whenever one factorial effect from an effects subspace occurs in $\boldsymbol{\beta}$, then all factorial effects of the effects subspace occur in $\boldsymbol{\beta}$.

Definition 2.2. A geometric factorial effects vector $\boldsymbol{\beta}$ will also be called hierarchical if whenever the factorial effects of one effects subspace occur in $\boldsymbol{\beta}$, then any factorial effects of the effects subspace involving the factors of any subset of the set consisting of all of the factors involved in this effects subspace also occur in $\boldsymbol{\beta}$.

\subsection{Orthogonal designs}

Suppose $\boldsymbol{\beta}$ is a vector of complex factorial effects that possesses the geometric property. We arrange the elements of $\gamma$ in such a way that 
$\boldsymbol{\gamma}=\left[\begin{array}{c}\boldsymbol{\beta}_{1} \\ \boldsymbol{\beta}_{2} \\ \vdots \\ \boldsymbol{\beta}_{h}\end{array}\right]$,

where each of the component vectors $\boldsymbol{\beta}_{1}, \boldsymbol{\beta}_{2}, \ldots, \boldsymbol{\beta}_{h}$ represents an entire effects subspace.

Further suppose a design $\boldsymbol{D}$ is used to collect data and the associated linear model is

$$
\boldsymbol{y}=\boldsymbol{X} \boldsymbol{\beta}+\boldsymbol{\varepsilon},
$$

where $\boldsymbol{y}$ is the observation vector corresponding to $\boldsymbol{D}, \boldsymbol{X}$ is the design matrix, and $\boldsymbol{\varepsilon}$ is an error vector with $V(\boldsymbol{\varepsilon})=\sigma^{2} \boldsymbol{I}$. The best linear unbiased estimator (BLUE) for $\boldsymbol{\beta}$ is $\hat{\boldsymbol{\beta}}=\left(\boldsymbol{X}^{\prime} \boldsymbol{X}\right)^{-1} \boldsymbol{X}^{\prime} \boldsymbol{y}$ and $V(\hat{\boldsymbol{\beta}})=\left(\boldsymbol{X}^{\prime} \boldsymbol{X}\right)^{-1} \sigma^{2}$. The design $\boldsymbol{D}$ is said to be orthogonal for $\boldsymbol{\beta}_{1}, \boldsymbol{\beta}_{2}, \ldots, \boldsymbol{\beta}_{h}$, if and only if the matrix $\boldsymbol{X}^{\prime} \boldsymbol{X}$ is block-diagonal where the blocks correspond to the subvectors of $\boldsymbol{\beta}$. What this means is, the estimator of any factorial effect in $\boldsymbol{\beta}$ is uncorrelated with the estimator of any other factorial effect in $\boldsymbol{\beta}$, provided they belong to different effects subspaces.

In practice, factorial effects may be defined by the analytic factorial effects which have useful interpretation (see Srivastava, 1990). It can be verified that if a design is orthogonal for a vector of complex factorial effects, then it is also orthogonal for the vector of analytic factorial effects consisting of the same effects subspaces (see Liao and Iyer, 1998).

\subsection{Parallel flats designs}

For $s_{1}^{n_{1}} \times s_{2}^{n_{2}} \times \cdots \times s_{g}^{n_{g}}$ mixed factorial experiments with $n_{1}+n_{2}+\cdots+n_{g}=n$, PFDs can be defined as follows. For $j=1,2, \ldots, f$, let $\boldsymbol{D}_{i j}$ consist of all solutions for $\boldsymbol{t}_{i}$ in the equation $\boldsymbol{A}_{i} \boldsymbol{t}_{i}^{\prime}=\boldsymbol{c}_{i j}$, where $\boldsymbol{A}_{i}$ is a $k_{i} \times n_{i}$ matrix of rank $k_{i}$ and $c_{i j}$ is a $k_{i} \times 1$ vector over $\mathrm{GF}\left(s_{i}\right)$, for $i=1,2, \ldots, g$. Then the single flat design $\boldsymbol{D}_{j}$ consists of all $\boldsymbol{t}=\left(\boldsymbol{t}_{1}, \boldsymbol{t}_{2}, \ldots, \boldsymbol{t}_{g}\right)$ with $\boldsymbol{t}_{i} \in \boldsymbol{D}_{i j}$, for $i=1,2, \ldots, g$.

Let $\boldsymbol{C}_{i}$ be the $k_{i} \times f$ matrix whose columns consist of the vectors $\boldsymbol{c}_{i 1}, \boldsymbol{c}_{i 2}, \ldots, \boldsymbol{c}_{i f}$, for $i=1,2, \ldots, g$. Then a PFD determined by the pair matrices $\left(\boldsymbol{A}_{i}, \boldsymbol{C}_{i}\right)$, for $i=1,2, \ldots, g$, is defined as the union of the $f$ single flat designs $\boldsymbol{D}_{1}, \boldsymbol{D}_{2}, \ldots, \boldsymbol{D}_{f}$. Clearly, such a design consists of $f \times s_{1}^{n_{1}-k_{1}} \times s_{2}^{n_{2}-k_{2}} \times \cdots \times s_{g}^{n_{g}-k_{g}}$ treatment combinations.

For $i=1,2, \ldots, g$, let $\boldsymbol{B}_{i}$ be any $n_{i} \times\left(n_{i}-k_{i}\right)$ matrix of rank $\left(n_{i}-k_{i}\right)$ over $\mathrm{GF}\left(s_{i}\right)$ such that $\boldsymbol{A}_{i} \boldsymbol{B}_{i}=\mathbf{0}$, where $\mathbf{0}$ is a matrix with all elements equal to 0 over $\operatorname{GF}\left(s_{i}\right)$. Also let $\boldsymbol{z}_{i j}=\left(z_{j 1}, \ldots, z_{j n_{i}}\right)^{\prime}$ be a particular solution of $\boldsymbol{A}_{i} \boldsymbol{t}_{i}^{\prime}=\boldsymbol{c}_{i j}$. Then the single flat design $\boldsymbol{D}_{j}$ consists of all $\boldsymbol{t}=\left(\boldsymbol{t}_{1}, \boldsymbol{t}_{2}, \ldots, \boldsymbol{t}_{g}\right)$ with $\boldsymbol{t}_{i} \in \boldsymbol{D}_{i j}$, where $\boldsymbol{D}_{i j}$ consists of all $\boldsymbol{t}_{i}$ satisfying

$$
\boldsymbol{t}_{i}^{\prime}=\boldsymbol{z}_{i j}+\boldsymbol{B}_{i} \boldsymbol{v}_{i}
$$

where $\boldsymbol{v}_{i}$ ranges over all possible vectors of length $\left(n_{i}-k_{i}\right)$ over $\mathrm{GF}\left(s_{i}\right)$ (there are $s_{i}^{n_{i}-k_{i}}$ such vectors). Therefore, a PFD can also be determined by the pair matrices $\left(\boldsymbol{B}_{i}, \boldsymbol{Z}_{i}\right)$, for $i=1,2, \ldots, g$, where $\boldsymbol{Z}_{i}$ is the $n_{i} \times f$ matrix whose columns consist of the vectors $\boldsymbol{z}_{i 1}, \boldsymbol{z}_{i 2}, \ldots, \boldsymbol{z}_{i f}$.

\section{Main theorem}

In this section we prove a necessary and sufficient condition for a PFD to be an orthogonal design for estimating a specified set of factorial effects $\boldsymbol{\beta}$ for $s_{1}^{n_{1}} \times s_{2}^{n_{2}} \times \cdots \times s_{g}^{n_{g}}$ experiments. In this paper, we only consider the case where $\boldsymbol{\beta}$ is geometric and hierarchical. Based on a linear model representation of complex factorial effects $\boldsymbol{\beta} ; \boldsymbol{y}=\boldsymbol{X} \boldsymbol{\beta}+\boldsymbol{\varepsilon}$ involving the elements of the matrix $\boldsymbol{X}^{\prime} \boldsymbol{X}$ have a convenient canonical form when the design used is a PFD. We state it in the following proposition. 
Proposition 3.1. Let $m\left[\boldsymbol{e}_{1}, \boldsymbol{e}_{2}\right]$ denote the element of the matrix $\boldsymbol{M}=\boldsymbol{X}^{\prime} \boldsymbol{X}$ corresponding to the row indexed by the factorial effect $\beta\left(\boldsymbol{e}_{1}\right)$ with $\boldsymbol{e}_{1}=\left(\boldsymbol{e}_{11}, \boldsymbol{e}_{12}, \ldots, \boldsymbol{e}_{1 g}\right)$ and the column indexed by $\beta\left(\boldsymbol{e}_{2}\right)$ with $\boldsymbol{e}_{2}=\left(\boldsymbol{e}_{21}, \boldsymbol{e}_{22}, \ldots, \boldsymbol{e}_{2 g}\right)$. Then

$$
m\left[\boldsymbol{e}_{1}, \boldsymbol{e}_{2}\right]=\left(\sum_{j=1}^{f} \prod_{i=1}^{g} w_{i}^{\left(\boldsymbol{e}_{1 i}+\boldsymbol{e}_{2 i}\right) z_{i j}}\right)\left(\prod_{i=1}^{g} \sum_{\boldsymbol{v}_{i}} w_{i}^{\left(\boldsymbol{e}_{1 i}+\boldsymbol{e}_{2 i}\right) \boldsymbol{B}_{i} \boldsymbol{v}_{i}}\right) .
$$

The pair matrices $\left(\boldsymbol{B}_{i}, \boldsymbol{Z}_{i}\right)$, for $i=1,2, \ldots, g$, determine a PFD; $w_{i}$ is a primitive $s_{i}$ th root of unity; and $\boldsymbol{v}_{i}$ ranges over all $s_{i}^{n_{i}-k_{i}}$ distinct vectors of length $\left(n_{i}-k_{i}\right)$ over $\operatorname{GF}\left(s_{i}\right)$.

Before proving the main theorem, we state the following lemmas whose proofs are straightforward.

Lemma 3.1. Let $\boldsymbol{x}$ be a nonzero vector over $\mathrm{GF}(s), s$ being a prime, of size $r \times 1$. Then the vector

$$
\boldsymbol{a}=\left(\boldsymbol{x}^{\prime} \boldsymbol{v}_{1}, \boldsymbol{x}^{\prime} \boldsymbol{v}_{2}, \ldots, \boldsymbol{x}^{\prime} \boldsymbol{v}_{s^{\prime}}\right)
$$

contains all elements of $\mathrm{GF}(s)$, each occurring $s^{r-1}$ times, where $\left\{\boldsymbol{v}_{1}, \boldsymbol{v}_{2}, \ldots, \boldsymbol{v}_{s^{r}}\right\}$ is the set of all $r \times 1$ vectors over $\mathrm{GF}(s)$.

Lemma 3.2. Let $m_{j}, j=0,1, \ldots, s-1$, be integers, and $w$ be a primitive sth root of unity, where $s$ is a prime. Then

$$
\sum_{j=0}^{s-1} m_{j} w^{k j}=0 \quad \text { for each } k=1,2, \ldots, s-1
$$

if and only if $m_{0}=m_{1}=\cdots=m_{s-1}$.

Lemma 3.3. Let $\boldsymbol{Q}$ be a $d \times f$ matrix whose $(i j)$ th element $q_{i j}$ is an element of $\mathrm{GF}\left(s_{i}\right)$, for $i=1,2, \ldots, d$ and $j=1,2, \ldots, f$, where $s_{1}, s_{2}, \ldots, s_{d}$ are primes. Then for any subset $\left\{i_{1}, i_{2}, \ldots, i_{h}\right\} \subseteq\{1,2, \ldots, d\}$, the following equation holds:

$$
\sum_{j=1}^{f}\left(w_{i_{1}}^{k_{i_{1}} q_{i_{1} j}} w_{i_{2}}^{k_{i_{2}} q_{i_{2} j}} \ldots w_{i_{h}}^{k_{i_{h}} q_{i_{h} j}}\right)=\sum_{j=1}^{f}\left(\prod_{l=1}^{h} w_{i_{l}}^{k_{i_{l}} q_{i_{l} j}}\right)=0 \text { for each } k_{i_{l}}=1,2, \ldots, s_{i_{l}}
$$

if and only if all possible $d \times 1$ vectors $\left(q_{1}, q_{2}, \ldots, q_{d}\right)^{\prime}$, where $q_{i}$ is an element of $\mathrm{GF}\left(s_{i}\right)$ occurs equal times as columns of $\boldsymbol{Q}$. Another way of stating this is that $\boldsymbol{Q}$ is an orthogonal array of strength $d$, briefly denoted by $\mathrm{OA}(d)$.

Suppose $\boldsymbol{\beta}$ consists of a specified set of factorial effects. We assume that $\boldsymbol{\beta}$ has the geometric and hierarchical properties and that any factorial effect not in the span of the elements of $\boldsymbol{\beta}$ is zero. For a given $\boldsymbol{\beta}$, we can define the following set $R$ :

$$
R=\left\{\begin{array}{l|c}
\left(\boldsymbol{e}_{11}+\boldsymbol{e}_{21}, \boldsymbol{e}_{12}+\boldsymbol{e}_{22}, \ldots, \boldsymbol{e}_{1 g}+\boldsymbol{e}_{2 g}\right) & \begin{array}{c}
\beta\left(\boldsymbol{e}_{11}, \boldsymbol{e}_{12}, \ldots, \boldsymbol{e}_{1 g}\right), \beta\left(\boldsymbol{e}_{21}, \boldsymbol{e}_{22}, \ldots, \boldsymbol{e}_{2 g}\right) \\
\text { are elements of } \boldsymbol{\beta} \text { and belong to distinct } \\
\text { effects subspaces }
\end{array}
\end{array} .\right.
$$

Now we prove a theorem giving a necessary and sufficient condition for a PFD to be orthogonal for $\boldsymbol{\beta}$. The PFD for mixed factorial experiments will be described in terms of pair matrices $\left(\boldsymbol{B}_{i}, \boldsymbol{Z}_{i}\right)$, for $i=1,2, \ldots, g$.

Theorem 3.1. In a $s_{1}^{n_{1}} \times s_{2}^{n_{2}} \times \cdots \times s_{g}^{n_{g}}$ experiment, with $s_{1}, s_{2}, \ldots, s_{g}$ being distinct primes, let a parallel flats design $\boldsymbol{D}$ be determined by the pair matrices $\left(\boldsymbol{B}_{i}, \boldsymbol{Z}_{i}\right)$, for $i=1,2, \ldots, g$. The factorial effects vector $\boldsymbol{\beta}$ is 
assumed to have geometric and hierarchical properties. For $\left(\boldsymbol{e}_{1}, \boldsymbol{e}_{2}, \ldots, \boldsymbol{e}_{g}\right) \in R$ defined as in Eq. (3.2), let $\left(\boldsymbol{e}_{i_{1}}, \boldsymbol{e}_{i_{2}}, \ldots, \boldsymbol{e}_{i_{d}}\right)$ be the vector consisting of nonzero subvectors of $\left(\boldsymbol{e}_{1}, \boldsymbol{e}_{2}, \ldots, \boldsymbol{e}_{g}\right)$. Then $\boldsymbol{D}$ is orthogonal for $\boldsymbol{\beta}$ if and only if $\boldsymbol{e}_{i_{l}} \boldsymbol{B}_{i_{l}}=\mathbf{0}$, for $l=1,2, \ldots, d$, imply that the array $\boldsymbol{Q}$ of size $d \times f$ given by

$$
\boldsymbol{Q}=\left[\begin{array}{c}
\boldsymbol{e}_{i_{1}} Z_{i_{1}} \\
\boldsymbol{e}_{i_{2}} Z_{i_{2}} \\
\vdots \\
\boldsymbol{e}_{i_{d}} Z_{i_{d}}
\end{array}\right]
$$

must be an $\mathrm{OA}(d)$.

Proof. Suppose $\beta\left(\boldsymbol{e}_{1}\right)$ with $\boldsymbol{e}_{1}=\left(\boldsymbol{e}_{11}, \boldsymbol{e}_{12}, \ldots, \boldsymbol{e}_{1 g}\right)$ and $\beta\left(\boldsymbol{e}_{2}\right)$ with $\boldsymbol{e}_{2}=\left(\boldsymbol{e}_{21}, \boldsymbol{e}_{22}, \ldots, \boldsymbol{e}_{2 g}\right)$ are in $\boldsymbol{\beta}$ and in different effects subspaces. By Proposition 3.1,

$$
m\left[\boldsymbol{e}_{1}, \boldsymbol{e}_{2}\right]=\left(\sum_{j=1}^{f} \prod_{i=1}^{g} w_{i}^{\left(\boldsymbol{e}_{1 i}+\boldsymbol{e}_{2 i}\right) z_{i j}}\right)\left(\prod_{i=1}^{g} \sum_{\boldsymbol{v}_{i}} w_{i}^{\left(\boldsymbol{e}_{1 i}+\boldsymbol{e}_{2 i}\right) \boldsymbol{B}_{i} \boldsymbol{v}_{i}}\right) .
$$

The elements $\left(\boldsymbol{e}_{11}+\boldsymbol{e}_{21}, \boldsymbol{e}_{12}+\boldsymbol{e}_{22}, \ldots, \boldsymbol{e}_{1 g}+\boldsymbol{e}_{2 g}\right)$ belong to the set $R$ defined in Eq. (3.2). Hence, $\boldsymbol{D}$ is orthogonal for $\boldsymbol{\beta}$ if and only if

$$
\left(\sum_{j=1}^{f} \prod_{i=1}^{g} w_{i}^{\boldsymbol{e}_{i} z_{i j}}\right)\left(\prod_{i=1}^{g} \sum_{\boldsymbol{v}_{i}} w_{i}^{\boldsymbol{e}_{i} \boldsymbol{B}_{i} \boldsymbol{v}_{i}}\right)=0 \quad \text { for all }\left(\boldsymbol{e}_{1}, \boldsymbol{e}_{2}, \ldots, \boldsymbol{e}_{g}\right) \in R,
$$

where $\boldsymbol{v}_{i}$ ranges over all $s_{i}^{n_{i}-k_{i}}$ distinct vectors of length $\left(n_{i}-k_{i}\right)$ over $\operatorname{GF}\left(s_{i}\right)$, for $i=1,2, \ldots, g$. From Lemmas 3.1 and 3.2, whenever one of $\boldsymbol{e}_{i} \boldsymbol{B}_{i} \neq \mathbf{0}$, for $\left(\boldsymbol{e}_{1}, \boldsymbol{e}_{2}, \ldots, \boldsymbol{e}_{g}\right) \in R$, Eq. (3.3) holds. Recall that $\boldsymbol{\beta}$ is assumed to be geometric and hierarchical. Thus, the necessary and sufficient condition follows directly from Lemma 3.3 .

We now give some examples to illustrate Theorem 3.1. The following array will be used to construct the examples:

$$
\boldsymbol{L}_{12}\left(2^{4} \cdot 3\right)=\left[\begin{array}{l}
\boldsymbol{l}_{1} \\
\boldsymbol{l}_{2} \\
\boldsymbol{l}_{3} \\
\boldsymbol{l}_{4} \\
\boldsymbol{l}_{5}
\end{array}\right]=\left[\begin{array}{llllllllllll}
0 & 0 & 1 & 1 & 0 & 0 & 1 & 1 & 0 & 0 & 1 & 1 \\
0 & 1 & 0 & 1 & 0 & 1 & 0 & 1 & 0 & 1 & 0 & 1 \\
0 & 0 & 1 & 1 & 1 & 1 & 0 & 0 & 1 & 0 & 0 & 1 \\
0 & 1 & 1 & 0 & 1 & 0 & 1 & 0 & 0 & 1 & 0 & 1 \\
0 & 0 & 0 & 0 & 1 & 1 & 1 & 1 & 2 & 2 & 2 & 2
\end{array}\right] .
$$

The reader can check that the above matrix $\boldsymbol{L}_{12}\left(2^{4} \cdot 3\right)$ is an OA(2) (see Wang and Wu, 1991). Also let $\boldsymbol{l}_{0}, \boldsymbol{l}_{6}$ and $\boldsymbol{l}_{7}$ be $1 \times 12$ vectors with all elements equal to 0 over $\operatorname{GF}(2), \operatorname{GF}(3)$, and $\operatorname{GF}(5)$, respectively.

Example 3.1. In a $2^{4} \times 3^{3}$ experiment, let $F_{1}, F_{2}, F_{3}$ and $F_{4}$ denote the four 2-level factors and $F_{5}, F_{6}$ and $F_{7}$ denote the three 3-level factors. Suppose $\boldsymbol{\beta}$ consists of the factorial effects belonging to the following effects subspaces:

$\mu ; F_{1}, F_{2}, F_{3}, F_{4}, F_{5}, F_{6}, F_{7} ; F_{1} F_{5}, F_{2} F_{5}, F_{3} F_{5}, F_{4} F_{5}$. 
It is assumed that any effect not in the span of these is zero. Let $\boldsymbol{B}_{1}=(0,0,0,0)^{\prime}, \boldsymbol{B}_{2}=(1,1,0)^{\prime}$,

$$
Z_{1}=\left[\begin{array}{l}
l_{1} \\
l_{2} \\
l_{3} \\
l_{4}
\end{array}\right] \quad \text { and } \quad Z_{2}=\left[\begin{array}{l}
l_{6} \\
l_{5} \\
l_{5}
\end{array}\right] .
$$

Using Theorem 3.1, it can be checked that the PFD determined by the matrices $\left(\boldsymbol{B}_{1}, \boldsymbol{Z}_{1}\right)$ and $\left(\boldsymbol{B}_{2}, \boldsymbol{Z}_{2}\right)$ is orthogonal for this $\boldsymbol{\beta}$ and has $12 \times 2^{0} \times 3^{3-2}=36$ runs.

Example 3.2. In a $2^{6} \times 3^{3}$ experiment, let $F_{1}, F_{2}, \ldots, F_{6}$ denote the six 2-level factors, and $F_{7}, F_{8}$ and $F_{9}$ denote the three 3-level factors. It can be checked that the PFD determined by $\boldsymbol{B}_{1}=(1,0,0,0,1,0)^{\prime}, \boldsymbol{B}_{2}=(1,1,0)^{\prime}$,

$$
Z_{1}=\left[\begin{array}{c}
\boldsymbol{l}_{0} \\
\boldsymbol{l}_{1} \\
\boldsymbol{l}_{2} \\
\boldsymbol{l}_{3} \\
\boldsymbol{l}_{4} \\
\boldsymbol{l}_{4}
\end{array}\right] \quad \text { and } \quad \boldsymbol{Z}_{2}=\left[\begin{array}{l}
\boldsymbol{l}_{6} \\
\boldsymbol{l}_{5} \\
\boldsymbol{l}_{5}
\end{array}\right]
$$

is orthogonal for $\boldsymbol{\beta}$ which consists of the factorial effects belonging to the following effects subspaces:

$$
\begin{gathered}
\mu ; F_{1}, F_{2}, F_{3}, F_{4}, F_{5}, F_{6}, F_{7}, F_{8}, F_{9} ; F_{1} F_{2}, F_{1} F_{3}, F_{1} F_{4}, F_{1} F_{7}, F_{1} F_{8}, \\
F_{2} F_{7}, F_{3} F_{7}, F_{4} F_{7}, F_{5} F_{7}, F_{5} F_{8} ; F_{1} F_{2} F_{7}, F_{1} F_{3} F_{7}, F_{1} F_{4} F_{7} .
\end{gathered}
$$

It is assumed that any effect not in the span of these is zero. This PFD has $12 \times 2^{6-5} \times 3^{3-2}=72$ runs.

Example 3.3. In a $2^{7} \times 3 \times 5^{2}$ experiment, let $F_{1}, F_{2}, \ldots, F_{7}$ denote the seven 2-level factors, $F_{8}$ denotes the 3-level factor, and $F_{9}$ and $F_{10}$ denote the two 5-level factors. Suppose $\boldsymbol{\beta}$ consists of the factorial effects belonging to the following effects subspaces:

$$
\begin{aligned}
\mu ; & F_{1}, F_{2}, F_{3}, F_{4}, F_{5}, F_{6}, F_{7}, F_{8}, F_{9}, F_{10} ; F_{1} F_{2}, F_{1} F_{3}, F_{1} F_{8}, F_{1} F_{9}, F_{1} F_{10}, \\
& F_{2} F_{9}, F_{2} F_{10}, F_{3} F_{9}, F_{3} F_{10}, F_{4} F_{9}, F_{4} F_{10}, F_{5} F_{9}, F_{5} F_{10}, F_{6} F_{9}, F_{6} F_{10}, \\
& F_{7} F_{9}, F_{7} F_{10}, F_{8} F_{9}, F_{8} F_{10} ; F_{1} F_{2} F_{9}, F_{1} F_{3} F_{10}, F_{1} F_{3} F_{9}, F_{1} F_{3} F_{10}, F_{1} F_{8} F_{9}, F_{1} F_{8} F_{10} .
\end{aligned}
$$

It is assumed that any effect not in the span of these is zero. Let $\boldsymbol{B}_{1}=(1,0,0,1,0,1,0)^{\prime}, \boldsymbol{B}_{2}=0, \boldsymbol{B}_{3}=(1,1)^{\prime}$,

$$
Z_{1}=\left[\begin{array}{l}
\boldsymbol{l}_{0} \\
\boldsymbol{l}_{1} \\
\boldsymbol{l}_{2} \\
\boldsymbol{l}_{3} \\
\boldsymbol{l}_{3} \\
\boldsymbol{l}_{4} \\
\boldsymbol{l}_{4}
\end{array}\right],
$$


$Z_{2}=\boldsymbol{l}_{5}$ and

$$
\boldsymbol{Z}_{3}=\left[\begin{array}{l}
\boldsymbol{l}_{7} \\
\boldsymbol{l}_{7}
\end{array}\right] .
$$

It can be checked that the PFD determined by the matrices $\left(\boldsymbol{B}_{i}, \boldsymbol{Z}_{i}\right)$, for $i=1,2,3$, is orthogonal for this $\boldsymbol{\beta}$ and has $12 \times 2^{7-6} \times 3^{0} \times 5^{2-1}=120$ runs.

\section{Concluding remarks}

Even though orthogonal designs constructed from the class of PFDs sometimes can have smaller run size than those constructed from the class of regular fractional factorial designs (single-flat designs) (see Liao et al., 1996). It can inevitably to lead to a very large run-size design due to the orthogonality requirement, particularly when the number of factors is large for the mixed factorial experiments. Therefore, it might be fruitful to investigate the construction of nearly orthogonal designs (see Wang and Wu, 1992) from the class of PFDs.

\section{References}

Connor, W.S., Young, S., 1961. Fractional Factorial Designs for Experiments with Factor at Two and Three Levels, Applied Mathematics Series. National Bureau of Standards, US Government Printing Office, Washington, DC.

Liao, C.T., Iyer, H.K., 1998. Orthogonal parallel-flats designs for $s_{1}^{n_{1}} \times s_{2}^{n_{2}}$ mixed factorial experiments. J. Statist. Plann. Inference 69, $175-191$.

Liao, C.T., Iyer, H.K., Vecchia, D.F., 1996. Construction of orthogonal two-level designs of user-specified resolution when $N \neq 2^{k}$. Technometrics. 38, 342-353.

Srivastava, J.N., 1987. Advances in the general theory of factorial designs based on partial pencils in Euclidean $n$-space. Utilitas Math. $32,75-94$.

Srivastava, J.N., 1990. Modern factorial design theory for experiments and statisticians. In: Ghosh, S. (Ed.), Statistical Design and Analysis in Industrial Experiments. Marcel Dekker, New York, pp. 311-401.

Srivastava, J.N., Anderson, D.A., Mardekian, J., 1984. Theory of factorial designs of parallel flat type. I. The coefficient matrix. J. Statist. Plann. Inference 9, 229-252.

Srivastava, J.N., Chopra, D.A., 1973. Balanced arrays and orthogonal arrays. In: Srivastava, J.N. (Ed.), A Survey of Combinatorial Theory. North-Holland, Amsterdam, pp. 411-428.

Srivastava, J.N., Li, J., 1996. Orthogonal designs of parallel-flats type. J. Statist. Plann. Inference 53, $261-283$.

Srivastava, J.N., Throop, D., 1990. Orthogonal arrays obtainable as solutions to linear equations over finite fields. Linear Algebra Appl. $127,283-300$.

Wang, J.C., Wu, C.F.J., 1991. An approach to the construction of asymmetrical orthogonal arrays. J. Amer. Statist. Assoc. 86, $450-455$.

Wang, J.C., Wu, C.F.J., 1992. Nearly orthogonal arrays with mixed levels and small runs. Technometrics 34, $409-422$. 